\title{
Locally optimal control of continuous-variable entanglement
}

\author{
Francesco Albarelli, ${ }^{1,2,3}$ Uther Shackerley-Bennett, ${ }^{3}$ and Alessio Serafini ${ }^{3}$ \\ ${ }^{1}$ Department of Physics, University of Warwick, Coventry CV4 7AL, United Kingdom \\ ${ }^{2}$ Quantum Technology Lab, Dipartimento di Fisica “Aldo Pontremoli”, Universitá degli Studi di Milano, I-20133 Milano, Italy \\ ${ }^{3}$ Department of Physics and Astronomy, University College London, Gower Street, London WC1E 6BT, United Kingdom
}

(Received 30 November 2017; revised manuscript received 14 June 2018; published 7 December 2018)

\begin{abstract}
We consider a system of two bosonic modes each subject to the dynamics induced by a thermal Markovian environment and we identify instantaneous, local symplectic controls that minimize the loss of entanglement in the Gaussian regime. By minimizing the decrease of the logarithmic negativity at every instant in time, it will be shown that a nontrivial, finite amount of local squeezing helps to counter the effect of decoherence during the evolution. We also determine optimal control routines in the more restrictive scenario where the control operations are applied on only one of the two modes. We find that applying an instantaneous control only at the beginning of the dynamics, i.e., preparing an appropriate initial state, is the optimal strategy for states with symmetric correlations and when the dynamics is the same on both modes. More generally, even in asymmetric cases, the delayed decay of entanglement resulting from the optimal preparation of the initial state with no further action turns out to be always very close to the optimized control where multiple operations are applied during the evolution. Our study extends directly to "monosymmetric" systems of any number of modes, i.e., to systems that are invariant under any local permutation of the modes within any one partition, as they are locally equivalent to two-mode systems.
\end{abstract}

DOI: 10.1103/PhysRevA.98.062312

\section{INTRODUCTION}

The property of entanglement exhibited by quantum systems has both metaphysical and technological interest. In applications, it has been found to be a fragile resource that is quickly lost when the system is not isolated. Since any manipulation of quantum systems requires them to be in contact with a noisy environment, a major question in advancing control upon them-arguably the main directive towards the development of functional quantum technologies-is how we can preserve this phenomenon for as long as it takes for an experiment to unfold. The design and application of quantum control techniques aimed at sustaining the entanglement of quantum systems has thus been a lively area of work over the past 15 years [1-17] that has seen the exploration of open-loop $[2,7]$ as well as measurement-based [3-6,8-10] and quantum coherent feedback [13] strategies, applicable in principle to a wide variety of systems, although quantum optical scenarios seem to offer accurate enough control and low enough noise to facilitate such endeavors $[1,11,16]$.

In this paper, we address the open-loop control of Gaussian entanglement, such as the one displayed in experiments based on parametric down conversion processes $[11,12,16]$. As well as providing an insightful theoretical landscape where, as we shall see, much can be evaluated analytically even when realistic noise is included, Gaussian systems are widely applicable not only to optical setups, but also in all other situations where the interaction between constituents and with the environment may be linearized, e.g., in optomechanics, ion traps, atomic ensembles, and certain quantum circuits [18]. In particular, we shall consider a two-mode system where each mode is subject to independent loss and thermal noise, as would be the case in nondegenerate parametric processes that give rise to two-mode squeezed states, the most representative of entangled Gaussian states [11,12]. Alongside the free, noisy evolution of an initial entangled state, which clearly degrades the entanglement, we shall consider the possibility of acting, at any instant in time, with arbitrary, impulsive local symplectic transformations. Let us remind the reader that such transformations, that will be assumed to be instantaneous, correspond to all local unitary operations that preserve the Gaussian character of the state. This assumption is not unreasonable if one considers, for instance, loss rates in the range of $10-10^{3} \mathrm{kHz}$ compared to manipulation times of the order of 1-10 ns, both achievable simultaneously in practice in a number of systems. This flavor of "optimal" control, whereby some figure of merit was locally optimized over instantaneous unitary manipulations, was already applied to discrete quantum systems in [19], was first extended to (single-mode) Gaussian states in [20], and was then adapted to the control of the global entropies of multimode Gaussian states in [21]. Here, we further extend this approach to the nontrivial question of controlling quantum entanglement of a two-mode system.

In this study, we will determine the optimal form of the local symplectic transformation that, at each given time, minimizes the loss in entanglement in terms of the logarithmic negativity, a suitable quantifier. Quite remarkably, we shall see that a single manipulation of the state through optimal local symplectic transformations is always optimal in cases with enough symmetry (either the same loss and thermal noise on both modes or initial states with symmetric correlations): in such cases no further impulsive transformations are required. It is worth mentioning that the application of a single initial 
Gaussian control to delay the loss of nonclassical properties of non-Gaussian states under the effect of decoherence has been considered previously in the literature [22-25]. Interestingly, we also have evidence that applying the control repeatedly only gives marginal improvements in the general case of asymmetric dynamics and correlations. Furthermore, we shall also consider the case where only one of the two modes can be controlled, showing that our analytical conditions extend to this case too.

There is, of course, no guarantee that this "time-local" optimal control, where the first derivative with respect to time of the figure of merit is optimized at all instants, would achieve a global optimization. Hence we shall test it against a different control strategy, where an alternative quantifier related to the entanglement of the evolving two-mode Gaussian state is optimized at all times. We find that the control based on the logarithmic negativity proves more robust: in our numerical investigation, we could find no way of outperforming it. We will also quantify the advantage our optimized control grants in certain practical cases, in terms of ebits of logarithmic negativity.

The paper is organized as follows.

\section{GAUSSIAN STATES}

We introduce here the necessary definitions and tools concerning Gaussian systems, following [18].

Let $\hat{\mathbf{r}}=\left(\hat{x}_{1}, \hat{p}_{1}, \ldots, \hat{x}_{n}, \hat{p}_{n}\right)^{\top}$ be a vector of canonical operators such that $\left[\hat{x}_{j}, \hat{p}_{k}\right]=i \delta_{j k}$, where $\delta_{j k}$ is the Kronecker delta. The canonical commutation relations may also be expressed as $\left[\hat{\mathbf{r}}, \hat{\mathbf{r}}^{\top}\right]=i \Omega$, where the commutator is to be interpreted as an outer product between vectors and the antisymmetric, symplectic form is given by

$$
\Omega=\bigoplus_{i=1}^{n}\left(\begin{array}{rr}
0 & 1 \\
-1 & 0
\end{array}\right)
$$

Second-order Hamiltonians are defined as those that can be written as $\hat{H}=\frac{1}{2} \hat{\mathbf{r}}^{\top} H \hat{\mathbf{r}}+\hat{\mathbf{r}}^{\top} \mathbf{a}$, where $H$ is a $2 n \times 2 n$, real, symmetric matrix and $\mathbf{a}$ is a vector of real numbers. The set of Gaussian states can be defined as the ground and thermal states of positive-definite quadratic Hamiltonians:

$$
\hat{\rho}_{G}=\frac{e^{-\beta \hat{H}}}{\operatorname{Tr}\left[e^{-\beta \hat{H}}\right]},
$$

where $\beta$ is the inverse temperature of the state. Note that the definition above encompasses the limiting instances of the inverse temperature $\beta$, which are needed to describe modes in pure quantum states. Such states are referred to as Gaussian due to their Wigner representation which takes a Gaussian form. As is well known, a Gaussian state $\hat{\rho}_{G}$ is completely characterized by the first- and second-order statistical moments of the canonical operators. First moments may be adjusted arbitrarily by local unitary "displacement" operations, and thus their values are inconsequential to the entanglement of a quantum state. We will therefore focus on the second moments alone, which are usually grouped together in the so-called covariance matrix:

$$
\boldsymbol{\sigma}=\operatorname{Tr}\left[\left\{(\hat{\mathbf{r}}-\mathbf{d}),(\hat{\mathbf{r}}-\mathbf{d})^{\top}\right\} \hat{\rho}_{G}\right],
$$

where $\mathbf{d}=\operatorname{Tr}\left[\hat{\mathbf{r}} \hat{\rho}_{G}\right]$ and the anticommutator is taken on the outer product of vectors of operators and the trace acts on the Hilbert space, thus obtaining a $2 n \times 2 n$ covariance matrix $\sigma$. In order to be a bona fide covariance matrix, $\sigma$ must abide by the uncertainty principle, $\sigma+i \Omega \geqslant 0$. In the following we will focus on two-mode Gaussian states; it is useful to express the $4 \times 4$ covariance matrix of the system in terms of $2 \times 2$ blocks as

$$
\sigma=\left(\begin{array}{cc}
\alpha & \gamma \\
\gamma^{\top} & \beta
\end{array}\right)
$$

where $\boldsymbol{\alpha}$ and $\boldsymbol{\beta}$ are the covariance matrices of the single-mode reduced states.

\section{Gaussian unitaries}

A unitary operation sends Gaussian states into Gaussian states if and only if it is generated by a second-order Hamiltonian. Since we are disregarding the first moments, we can set $\mathbf{a}=0$ in the definition above and thus obtain the group of symplectic transformations, generated at the Hilbert space level by purely quadratic Hamiltonians. A symplectic transformation $S$ describes the linear, Heisenberg-picture evolution of the vector $\hat{\mathbf{r}}$ under such a purely quadratic Hamiltonian as $\hat{\mathbf{r}} \mapsto S \hat{\mathbf{r}}$. Since they correspond to unitary operations, symplectic transformations must preserve the canonical commutation relations: they can in fact be defined as the set of $2 n \times 2 n$ matrices $S$ such that

$$
S \Omega S^{\top}=\Omega
$$

which form the real symplectic group $\mathrm{Sp}_{2 n, \mathbb{R}}$. Covariance matrices transform under the finite-dimensional representation of the symplectic group by conjugation:

$$
\sigma \mapsto S \sigma S^{\top}
$$

In the study at hand, we will focus on two-mode states and consider control strategies enacted through local symplectic transformations, acting separately on each mode, which belong to the direct sum $\mathrm{Sp}_{2, \mathbb{R}} \oplus \mathrm{Sp}_{2, \mathbb{R}}$. Explicitly, a generic local symplectic transformation $S_{\text {loc }}$ on two modes may be written as

$$
S_{\mathrm{loc}}=\left(\begin{array}{cc}
S_{1} & 0 \\
0 & S_{2}
\end{array}\right), \quad \text { with } S_{1}, S_{2} \in \mathrm{Sp}_{2, \mathbb{R}} .
$$

In the quantum optical practice, such operations correspond to sequences of single-mode squeezers and phase shifters (also known as "phase plates").

Many properties of Gaussian states can be described in terms of symplectic invariants, quantities invariant under symplectic transformations, which we present more in detail in Appendix A. On the other hand, the entanglement of Gaussian states can be discussed in term of local symplectic invariants; this topic is discussed in Appendix B, along with separability conditions and entanglement quantifiers for Gaussian states.

\section{OPTIMAL TIME-LOCAL CONTROL}

\section{A. Free evolution}

As the free, uncontrolled, evolution of the system, responsible for the loss of coherence and entanglement, we 
shall adopt the rotating wave interaction of the system with a number of bosonic Markovian environments with a different coupling strength and number of thermal excitations for each mode. The evolution of the state is described the following Lindblad master equation:

$$
\dot{\hat{\rho}}=\sum_{i=1}^{n} \gamma_{i}\left(\bar{N}_{i}+1\right) \mathcal{D}\left[\hat{a}_{i}\right] \hat{\rho}+\gamma_{i} \bar{N}_{i} \mathcal{D}\left[\hat{a}_{i}^{\dagger}\right] \hat{\rho},
$$

where the superoperator $\mathcal{D}[\hat{o}] \hat{\rho}=\hat{o} \hat{\rho} \hat{o}^{\dagger}-\left\{\hat{o}^{\dagger} \hat{o}, \hat{\rho}\right\}$ was introduced together with the usual annihilation operators $\hat{a}_{k}=$ $\left(\hat{x}_{k}+i \hat{p}_{k}\right) / \sqrt{2}$. This dynamics is often referred to as "loss in a thermal environment," in this case a different thermal environment for each mode. The parameters $\gamma_{i}$ represent the loss rates (we could omit one by scaling the unit of time), whilst $\bar{N}_{i}=1 /\left(\exp \left[\frac{\hbar \omega_{i}}{k T_{i}}\right]-1\right)$ are the mean number of bosonic excitations of each bath, written in terms of the baths' temperatures $T_{i}$, the modes' frequencies $\omega_{i}$, Planck's constant $\hbar$, and Boltzmann's constant $k$. We remark that the number of excitations $\bar{N}_{i}$ are different for bosonic modes at different frequencies even if the temperature is the same. This is relevant in various physical setups, e.g., quantum states of the electromagnetic field generated by nondegenerate parametric down conversion or analogous processes [26].

In terms of the covariance matrix, this dynamics leads to a diffusive equation [18]:

$$
\dot{\sigma}=A \sigma+\sigma A^{\top}+D
$$

with $A=-\frac{1}{2} \bigoplus_{i}^{n} \gamma_{i} \mathbb{1}_{2}$ and $D=\bigoplus_{i}^{n} \chi_{i} \mathbb{1}_{2}$ and $\chi_{i}=$ $\gamma_{i}\left(2 N_{i}+1\right)$. Throughout the paper, the notation $\mathbb{1}_{j}$ will indicate the $j \times j$ identity matrix. We remark that Eq. (9) holds true for the covariance matrix of any state (regardless of its Gaussian character) undergoing the dynamics given by Eq. (8). To completely determine the evolution of a Gaussian state, one also needs the equation for the first moments $\dot{\mathbf{d}}=$ Ad. However, we will only need the evolution of the covariance matrix, since it encodes all the entanglement properties of a Gaussian state.

\section{B. Local control}

We will now assume the possibility of performing instantaneous, local symplectic transformations, as given by Eq. (7), which cannot generate quantum correlations but can, as we shall see, delay their decay during the interaction with the thermal bath described by the diffusive dynamics (9).

To start with, observe that, since we are assuming arbitrary local symplectic control, as in Eq. (7), we can restrict to covariance matrices in Simon normal form, which can be reached through local symplectic transformations [18,27]; the blocks of a covariance matrix $\sigma_{\mathrm{nf}}$ in normal form are all diagonal:

$$
\boldsymbol{\alpha}_{\mathrm{nf}}=a \mathbb{1}_{2}, \quad \boldsymbol{\beta}_{\mathrm{nf}}=b \mathbb{1}_{2}, \quad \boldsymbol{\gamma}_{\mathrm{nf}}=\operatorname{diag}\left(c_{+}, c_{-}\right) .
$$

Here, $a, b, c_{+}$, and $c_{-}$may be taken as the four independent local symplectic invariants, in terms of which the optimization problem we set to solve may be cast. Such invariants may be promptly related to a set of quantities, such as Det $\sigma$, Det $\boldsymbol{\alpha}$, $\operatorname{Det} \boldsymbol{\beta}$, and Det $\boldsymbol{\gamma}$, whose invariance is manifest since any local symplectic matrix $S$ has $\operatorname{Det} S=1$. Since local rotations are symplectic transformations that preserve the local covariance matrices, we may swap $c_{+}$and $c_{-}$and without loss of generality assume $c_{+} \geqslant c_{-}$.

Our main result is the analytic expression of the optimal separable Gaussian unitary which maximizes the derivative of the logarithmic negativity under the dynamics of Eq. (9). The optimal control unitary is given by two local squeezing operations $S_{j}=\operatorname{diag}\left(z_{j}, 1 / z_{j}\right)[18,28]$; the optimal squeezing parameters are

$$
\bar{z}_{j}=\sqrt[4]{\frac{w_{j}}{v_{j}}},
$$

where we introduced the coefficients

$$
\begin{aligned}
& v_{1}=a\left(b^{2}-a^{2}+u\right)+2 c_{+}\left(a c_{-}-b c_{+}\right), \\
& w_{1}=a\left(b^{2}-a^{2}+u\right)+2 c_{-}\left(a c_{+}-b c_{-}\right), \\
& v_{2}=b\left(a^{2}-b^{2}+u\right)+2 c_{+}\left(b c_{-}-a c_{+}\right), \\
& w_{2}=b\left(a^{2}-b^{2}+u\right)+2 c_{-}\left(b c_{+}-a c_{-}\right),
\end{aligned}
$$

which in turn depend on the parameter

$u=\sqrt{\left(a^{2}-b^{2}\right)^{2}+4 a b\left(c_{+}^{2}+c_{-}^{2}\right)-4 c_{+} c_{-}\left(a^{2}+b^{2}\right)}$.

Furthermore, if only one of the two modes can be acted on, the optimal control operation is still a (single) local squeezing with the same coefficient reported in Eq. (11).

In the next subsection we shall present the derivation of these results.

\section{Derivation of the optimal local squeezing}

The logarithmic negativity [29] is the entanglement quantifier that we shall adopt as a figure of merit for our control scheme. For a two-mode Gaussian state it can be computed as

$$
E_{\mathcal{N}}=\max \left\{0,-\log _{2}\left(\tilde{v}_{-}\right)\right\}
$$

where $\tilde{v}_{-}$is the smallest symplectic eigenvalue of the partially transposed state, which reads

$$
2 \tilde{v}_{-}^{2}=\tilde{\Delta}_{1}^{2}-\sqrt{\left(\tilde{\Delta}_{1}^{2}\right)^{2}-4 \operatorname{Det} \sigma},
$$

where $\tilde{\Delta}_{1}^{2}=\operatorname{Det} \boldsymbol{\alpha}+\operatorname{Det} \boldsymbol{\beta}-2 \operatorname{Det} \boldsymbol{\gamma}$. For a more thorough treatment of the subject, see Appendix B.

Our control objective is thus to minimize the derivative of $2 \tilde{\nu}_{-}^{2}$, since the logarithmic negativity $E_{\mathcal{N}}$ is a decreasing function of it. It is thus useful to work out explicitly its derivatives with respect to Deto and $\tilde{\Delta}_{1}^{2}$ :

$$
\begin{aligned}
\frac{\partial 2 \tilde{\nu}_{-}^{2}}{\partial \operatorname{Det} \sigma} & =\frac{2}{\sqrt{\left(\tilde{\Delta}_{1}^{2}\right)^{2}-4 \operatorname{Det} \sigma}}=\frac{2}{u}, \\
\frac{\partial 2 \tilde{v}_{-}^{2}}{\partial \tilde{\Delta}_{1}^{2}} & =1-\frac{\tilde{\Delta}_{1}^{2}}{\sqrt{\left(\tilde{\Delta}_{1}^{2}\right)^{2}-4 \operatorname{Det} \sigma}} \\
& =1-\frac{a^{2}+b^{2}-2 c_{+} c_{-}}{u} .
\end{aligned}
$$


The quantity $u=\sqrt{\left(\tilde{\Delta}_{1}^{2}\right)^{2}-4 \operatorname{Det} \sigma}$ is reported as a function of the normal form coefficients in Eq. (16); notice that it is always strictly greater than zero for entangled states. To see this, it will suffice to notice that only one partially transposed symplectic eigenvalue of a two-mode covariance matrix may ever be smaller than 1 [18,30], as is the case for an entangled state. Therefore, the two symplectic eigenvalues of a twomode entangled state must be different, which is equivalent to stating $u>0$.

Now, the free, diffusive evolution of the quantities Deto and $\tilde{\Delta}_{1}^{2}$ can be written as (see Appendix $\mathrm{C}$ for the derivation)

$$
\begin{aligned}
\operatorname{Det} \boldsymbol{\sigma}= & -2\left(\gamma_{1}+\gamma_{2}\right) \operatorname{Det} \boldsymbol{\sigma}+\chi_{1} \operatorname{Det} \boldsymbol{\beta} \operatorname{Tr}\left[S_{1}(\boldsymbol{\sigma} / \boldsymbol{\beta}) S_{1}^{\top}\right] \\
& +\chi_{2} \operatorname{Det} \boldsymbol{\alpha} \operatorname{Tr}\left[S_{2}(\boldsymbol{\sigma} / \boldsymbol{\alpha}) S_{2}^{\top}\right] \\
\dot{\tilde{\Delta}}_{1}^{2}= & -2 \gamma_{1} \operatorname{Det} \boldsymbol{\alpha}-2 \gamma_{2} \operatorname{Det} \boldsymbol{\beta}+2\left(\gamma_{1}+\gamma_{2}\right) \operatorname{Det} \boldsymbol{\gamma} \\
& +\chi_{1} \operatorname{Tr}\left[S_{1} \boldsymbol{\alpha} S_{1}^{\top}\right]+\chi_{2} \operatorname{Tr}\left[S_{2} \boldsymbol{\beta} S_{2}^{\top}\right]
\end{aligned}
$$

where $\boldsymbol{\sigma} / \boldsymbol{\beta}=\boldsymbol{\alpha}-\boldsymbol{\gamma} \boldsymbol{\beta}^{-1} \boldsymbol{\gamma}^{\top}$ and $\boldsymbol{\sigma} / \boldsymbol{\alpha}=\boldsymbol{\beta}-\boldsymbol{\gamma}^{\top} \boldsymbol{\alpha}^{-1} \boldsymbol{\gamma}$ are the Schur complements of $\boldsymbol{\sigma}$ with respect to the submatrices $\boldsymbol{\beta}$ and $\boldsymbol{\alpha}$. Here, the role of the control operation has been made explicit through the local symplectic transformations $S_{1}$ and $S_{2}$. We intend to determine the symplectic matrices $S_{1}$ and $S_{2}$ that minimize the derivative of $\tilde{v}_{-}$and thus optimally preserve the entanglement over an infinitesimal time interval, whereby our control is termed "local" or "time local."

Each of the single-mode symplectic matrices $S_{1}$ and $S_{2}$ admits a singular value decomposition as $S_{j}=Q_{j} Z_{j} R_{j}$, where $Q_{j}$ and $R_{j}$ are orthogonal symplectic matrices and $Z_{j}=\operatorname{diag}\left(z_{j}, 1 / z_{j}\right)$ is a local squeezing operation. This fact simplifies our optimization considerably: first, notice that all the terms depending on $S_{j}$ in Eqs. (21) and (22) are invariant under local orthogonal transformations, so that the transformations $Q_{j}$ can be ignored altogether. Besides, due to our use of the Simon normal form it may be shown that the minimization we are considering below is always realized for $R_{j}=\mathbb{1}_{2}$ and a proper choice of $Z_{j}$. Hence the only relevant local action may come from the local squeezing operations $Z_{1}$ and $Z_{2}$, diagonal in the normal quadratures (as we shall refer to the degrees of freedom that attain the Simon normal form).

The straightforward evaluation of the quantities depending on the local symplectic transformations in Eqs. (21) and (22) yields

$$
\operatorname{Det} \boldsymbol{\beta} \operatorname{Tr}\left[S_{1}(\boldsymbol{\sigma} / \boldsymbol{\beta}) S_{1}^{\top}\right]=\left[\frac{b\left(a b-c_{-}^{2}\right)}{z_{1}^{2}}+b\left(a b-c_{+}^{2}\right) z_{1}^{2}\right] \text {, }
$$

$$
\begin{aligned}
\operatorname{Det} \boldsymbol{\alpha} \operatorname{Tr}\left[S_{2}(\boldsymbol{\sigma} / \boldsymbol{\alpha}) S_{2}^{\top}\right]=\left[\frac{a\left(a b-c_{-}^{2}\right)}{z_{2}^{2}}+a\left(a b-c_{+}^{2}\right) z_{2}^{2}\right] \\
\operatorname{Tr}\left[Z_{1} \boldsymbol{\alpha} Z_{1}^{\top}\right]=a\left(z_{1}^{2}+\frac{1}{z_{1}^{2}}\right) \\
\operatorname{Tr}\left[Z_{2} \boldsymbol{\beta} Z_{2}^{\top}\right]=b\left(z_{2}^{2}+\frac{1}{z_{2}^{2}}\right)
\end{aligned}
$$

Now, our optimization over the squeezing parameters $z_{1}$ and $z_{2}$ may be defined by considering only the additive part in the derivative of $\left(2 \tilde{v}_{-}\right)^{2}$ that contains such parameters; it will also be convenient to multiply such a term by the positive quantity $u>0$ (that does not depend on $z_{1}$ and $z_{2}$ ) and to refer to the quantity obtained by $\xi$. Equations (19),(20) and (23)-(26) yield

$$
\xi=\chi_{1}\left(v_{1} z_{1}^{2}+\frac{w_{1}}{z_{1}^{2}}\right)+\chi_{2}\left(v_{2} z_{2}^{2}+\frac{w_{2}}{z_{2}^{2}}\right)
$$

All of the quantities $v_{j}$ and $w_{j}$, defined in Eqs. (12)-(15), are bound to be positive semidefinite: if that were not the case, there would exist physical cases where the free dynamics, that corresponds to local completely positive maps, would be able to increase the logarithmic negativity, which is impossible since the latter is an entanglement monotone [29]. In point of fact, it will be expedient to just consider the quantities $v_{j}$ and $w_{j}$ as strictly positive in what follows. The possibility for such parameters to take null values would imply the existence of limiting states where the free, thermal lossy channel does not decrease the entanglement: we are not aware of any such states (nor did we encounter any numerical evidence where any of the parameters above were zero).

Hence the minimization of $\xi$, which yields the optimal local squeezing parameters to preserve as much quantum entanglement as possible (in the form of logarithmic negativity), is performed by separately minimizing the two terms in (27), as they are both strictly positive; the dependence on the two parameters $\chi_{j}$ thus vanishes. The result is straightforward and furnishes the optimal parameters in Eq. (11).

\section{Time-local control of a single subsystem}

We now consider a different scenario where we have the ability to control only one of the two subsystems; without loss of generality we assume this to be the first subsystem, the one corresponding to the local covariance matrix $\alpha$. Differently from the previous case we cannot put the state in normal form, since local symplectic transformations on both modes would be needed. We can however apply symplectic transformations on one system to make the local covariance matrix $\boldsymbol{\alpha}$ proportional to the identity, and then further act on such a subsystem with a local rotation (which will leave $\boldsymbol{\alpha}$ unaffected but can act on the correlation sub-block $\gamma$ ). In this case, the free parameters are eight, and it is convenient to parametrize the whole covariance matrix in terms of the coefficients of the normal form as follows:

$$
\sigma=S_{\alpha, \beta} \sigma_{\mathrm{nf}} S_{\alpha, \beta}^{\top}, \quad S_{\alpha, \beta}=\mathbb{1}_{2} \oplus S_{\beta}
$$

$S_{\beta}$ is a generic symplectic transform on the second subsystem. We can readily see that the ten free real parameters of a generic two-mode covariance matrix are reduced to eight, since $S_{\beta}$ will depend on two angles and a (unbounded) squeezing parameter.

We can then reproduce all the previous steps; in particular, we now have to evaluate Eqs. (21) and (22) considering only the local symplectic control $S_{1}=Z_{1} R_{1}$; i.e., setting $S_{2}=$ $\mathbb{1}_{2}$. The minimization proceeds in the same way, with the minimum always attained for $R_{1}=\mathbb{1}_{2}$ and for a diagonal squeezing operation $Z_{1}$ with squeezing parameter $\bar{z}_{1}$ given by 
Eq. (11) in terms of the coefficients of the normal form. Even if the state cannot be put in normal form, the only parameters that come into play are the normal form coefficients, since the local symplectic $S_{\beta}$ on the initial state does not affect the result. This is due to the fact that, despite the presence of entanglement, the contributions of the individual modes to the derivative in Eqs. (21) and (22) are positive and independent. Note that the symplectic transformation $S_{1}$ is the control operation, whilst $S_{\beta}$ above was only used to parametrize the initial state.

\section{Initial-state preparation versus repeated control operations}

A few preliminary considerations concerning the optimal control strategy emerge directly from Eqs. (11)-(15). If the magnitudes of the $x$ and $p$ correlations in the normal form $c_{+}$and $c_{-}$are the same, i.e., if $c_{+}=-c_{-}$(note that $c_{+}$and $c_{-}$of different signs are a prerequisite for entanglement [18]), then $v_{j}=w_{j}$ and $\bar{z}_{j}=1$ for $j=1,2$. The optimal control strategy consists therefore just in putting the initial state in normal form through local symplectic transformations, and then in letting the state evolve freely, since the evolution we are considering will keep the covariance matrix in normal form. In the following we refer to this condition $\left|c_{+}\right|=\left|c_{-}\right|$ by saying that the state has "symmetric correlations."

However, if $\left|c_{+}\right| \neq\left|c_{-}\right|$, then the optimal control strategy includes some local squeezing after the normal form reduction; the form of such a locally squeezed state is not preserved by the dynamics. Nonetheless, when the two modes undergo the same dynamics, i.e., $\gamma_{1}=\gamma_{2}$ and $\chi_{1}=\chi_{2}$, it turns out that a single iteration of the initial control operation leads to timelocal optimal control over the whole dynamics. To write this in formulas we consider a given optimal covariance matrix $\sigma^{\prime}$, i.e., $\sigma^{\prime}=S_{\mathrm{loc}} \sigma_{0} S_{\mathrm{loc}}^{\top}$, such that $S_{\mathrm{loc}}$ minimizes $\dot{\tilde{v}}\left(S_{\mathrm{loc}} \sigma_{0} S_{\mathrm{loc}}^{\top}\right)$ and $\sigma_{0}$ is a completely generic initial physical covariance matrix. The statement above is then equivalent to

$$
\inf _{S_{\mathrm{loc}}} \dot{\tilde{v}}\left(S_{\mathrm{loc}}\left[p \boldsymbol{\sigma}^{\prime}+(1-p) \chi \mathbb{1}_{4}\right] S_{\mathrm{loc}}^{\top}\right)=\dot{\tilde{v}}\left[p \boldsymbol{\sigma}^{\prime}+(1-p) \chi \mathbb{1}_{4}\right],
$$

$\forall p \in[0,1]$, with $S_{\text {loc }}$ optimized over the set $\mathrm{Sp}_{2, \mathbb{R}} \oplus \mathrm{Sp}_{2, \mathbb{R}}$. The convex combination appearing in the previous equation is the diffusive time evolution obtained by solving Eq. (9) with the choice $\gamma_{i}=\gamma, \chi_{i}=\chi$, a rescaling of time $t \rightarrow \gamma t$, and upon the substitution $p=e^{-t}$ [see Eqs. (C2)-(C4) for the general case]. The statement above is proven by showing that the local squeezing of the matrix $p \boldsymbol{\sigma}^{\prime}+(1-p) \chi \mathbb{1}_{4}$ matches, for all $p$, the optimal squeezing condition (11), where the quantities (12)-(15) must be reevaluated for $p \boldsymbol{\sigma}^{\prime}+(1-p) \chi \mathbb{1}_{4}$. This yields an equality in terms of the quantities $a, b, c_{+}$, and $c_{-}$of the initial state, whose explicit algebraic expression is utterly unwieldy, but which can be verified exactly with Mathematica.

In general, when $\chi_{1} \neq \chi_{2}$ and $\gamma_{1} \neq \gamma_{2}$ the previous statement does not hold. Therefore, we can quantitatively evaluate the effectiveness of repeated control operations applied during the considered dynamics. Numerical evidence (presented in Sec. IV) shows that subsequent control operations after the initial one improve the conservation of entanglement, but by a very small amount. The intuitive reason behind this behavior is that the repeated scheme gets more useful the greater the unbalance between the values $\left|c_{+}\right|$and $\left|c_{-}\right|$. However, an unbalance between those correlation terms is achieved only for mixed states and, due to the uncertainty principle that restricts physical covariance matrices, there is only a narrow region in parameter space where such unbalanced states contain substantial entanglement [31].

\section{APPLICATIONS}

Here, we shall present some quantitative applications of the results we have derived. We do not concentrate on a specific physical platform in great detail, but the choice of the parameters $\gamma_{i}$ and $\bar{N}_{i}$ in the following are apt to describe quantum states of the electromagnetic field in the optical (Sec. IV A) and microwave domain (Sec. IV B).

\section{A. Optimal state preparation}

As previously stated, in cases with enough symmetry the only control operation needed is an appropriate state preparation. Let us first consider the paradigmatic example of an initial two-mode squeezed state, with $a=b=\cosh (2 r)$ and $c_{+}=-c_{-}=\sinh (2 r), r$ being the so-called two-mode squeezing parameter. Such states are the output of nondegenerate parametric down conversion processes, and possibly the most iconic among entangled Gaussian states. For a state with symmetric correlations the optimal control operation is to bring it in normal form; therefore, a two-mode squeezed state is already optimal. We study the dynamics of the logarithmic negativity for initial states that differ from a two-mode squeezed state by a local squeezing operation $\operatorname{diag}(2,1 / 2)$, applied on only one mode or applied on both modes. The first case can also be thought of as a single-mode control scenario, as described in Sec. III B 2.

The advantage granted by the initial adjustment is illustrated in Fig. 1, where equal loss rates and equal thermal noises are assumed for both modes. In the case of high thermal noise $\chi / \gamma=2$ (left panel of Fig. 1) it is apparent that the

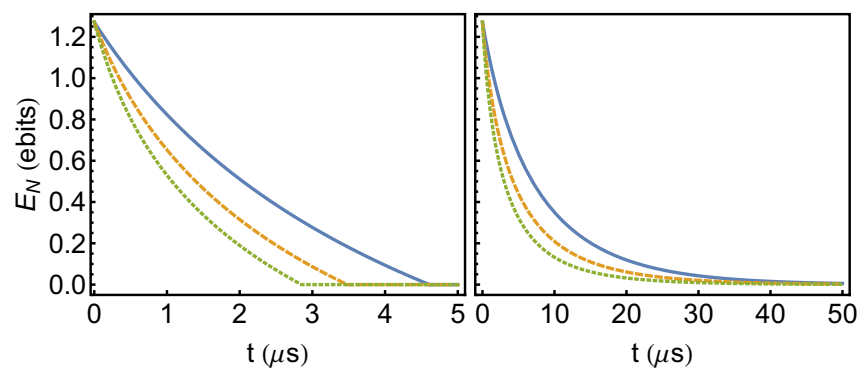

FIG. 1. Logarithmic negativity of states which are initially locally equivalent to a pure two-mode squeezed state with squeezing parameter $r=\ln [\sqrt{2}+1] / 2$, evolving in a thermal environment with $\chi / \gamma=2$ (left panel) and $\chi / \gamma=1.000013$, i.e., room temperature at $450 \mathrm{THz}$ (right panel); the two modes have the same loss rate $\gamma=100 \mathrm{kHz}$. The blue (solid) curve refers to an initial state in standard form and the orange (dashed) curve refers to an initial state where one mode is locally squeezed by the squeezing transformation $\bar{Z}=\operatorname{diag}(2,1 / 2)$, while the green (dotted) curve refers to a state where both modes have been squeezed by $\bar{Z}$. 


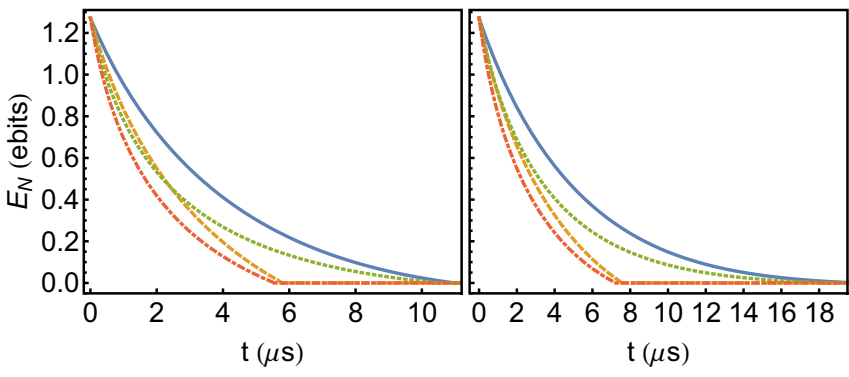

FIG. 2. Logarithmic negativity of states which are initially locally equivalent to a pure two-mode squeezed state with squeezing parameter $r=\ln [\sqrt{2}+1] / 2$. Left panel: the two modes are evolving in two thermal environments with $\chi_{1} / \gamma=1$ and $\chi_{2} / \gamma=2$, while the loss rate $\gamma=100 \mathrm{kHz}$ is the same for both modes. Right panel: the two modes have different loss rates $\gamma_{1}=100 \mathrm{kHz}$ and $\gamma_{2}=10 \mathrm{kHz}$, but the same parameter $\chi=\gamma_{1} 1.000013$. The blue (solid) curve refers to an initial state in standard form, the orange (dashed) curve refers to an initial state where the first mode is locally squeezed by the squeezing transformation $\bar{Z}=\operatorname{diag}(2,1 / 2)$, and the green (dotted) curve refers to a state where the second mode is squeezed by $\bar{Z}$, while the red (dot-dashed) curve refers to a state where both modes have been squeezed by $\bar{Z}$.

initial control may prolong the life of quantum entanglement by around $2 \mu$ s (this will of course depend on the strength of the single-mode squeezing operation considered). Reducing $\chi / \gamma$ to 1.000013 , which corresponds to the roomtemperature thermal noise affecting a mode of visible radiation at $450 \mathrm{THz}$, results in the comparison in the right panel of Fig. 1: as is well known, at such low noise the complete demise of entanglement is less sharp, but adjusting the state in normal form still allows one to gain about 0.22 ebits of logarithmic negativity after $10 \mu \mathrm{s}$ : a very significant improvement.

Figure 2 shows the effect of different decoherence channels on the same initial two-mode squeezed vacuum. In the left panel we keep the same loss rate $\gamma=100 \mathrm{kHz}$ but we change the thermal noise affecting the two modes $\left(\chi_{1} / \gamma=2\right.$ and $\left.\chi_{2} / \gamma=1\right)$, while in the right panel we consider two different loss rates $\gamma_{1}=100 \mathrm{kHz}$ and $\gamma_{2}=10 \mathrm{kHz}$, but we keep the same parameter $\chi=\gamma_{1} 1.000013$. In this case it becomes evident how the symmetry between the two modes is lost and depending on which one of the two modes is controlled we have different behaviors.

Figure 3 illustrates the effect of a single control step in instances relevant to optical systems, and highlights the influence local operations might have on the dynamics of entanglement under decoherence. In the case depicted, which adopts as above a loss rate of $100 \mathrm{kHz}$ and $\chi=1.000013$ (room temperature at $450 \mathrm{THz}$ ), the so-called "sudden death" of quantum entanglement is delayed from $7 \mu$ s to a stunning $90 \mu$ s though the optimal control squeezing operation identified above. It is worth stressing again that the optimal local squeezing transformation does not depend on the parameters of the noise, but only on the state in hand.

\section{B. Repeated controls}

As explained previously, repeatedly applying the control during the dynamics is only relevant for initial states with un-

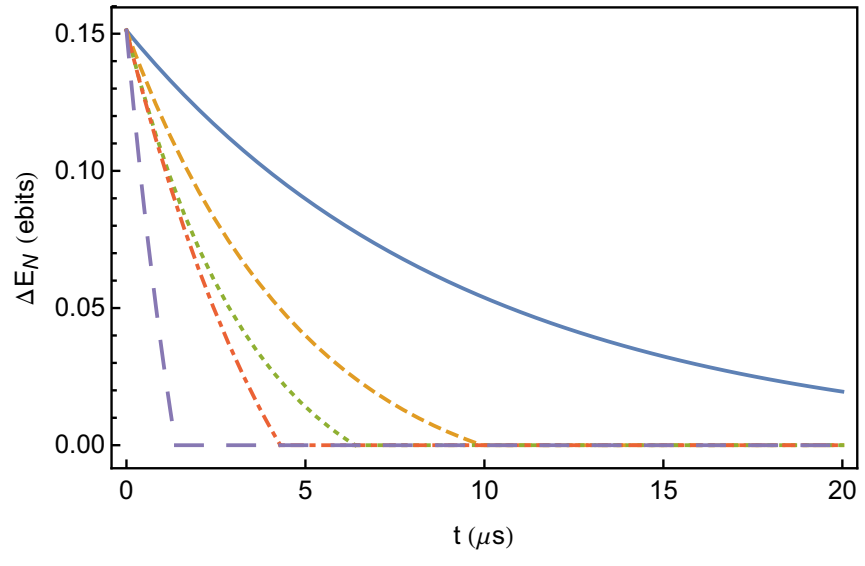

FIG. 3. Logarithmic negativity for initial states locally equivalent to a state with normal form parameters $a=4.5, b=3.5, c_{+}=2.2$, and $c_{-}=-3.5$, evolving in a thermal environment with $\chi / \gamma=$ 1.000013 and loss rate $\gamma=100 \mathrm{kHz}$. The blue (solid) curve pertains to a state that was optimally adjusted on both modes, the orange (short dashed) curve pertains to a state that was optimally adjusted on one mode, the green (dotted) curve pertains to an initial state in normal form, and the red (dot-dashed) curve pertains to an initial state whose normal form was altered by the squeezing transformation $\bar{Z}=\operatorname{diag}(2,1 / 2)$ on one mode, while the purple (large dashed) curve pertains to a state further altered locally by a phase plate $R_{\frac{\pi}{4}}$ (besides the local squeezing $\bar{Z}$ ).

balanced correlations $\left|c_{+}\right| \neq\left|c_{-}\right|$and if the reduced dynamics of the two modes is not the same. For this quantitative study, we therefore choose thermal environments corresponding to different microwave frequencies of $\omega_{1}=35.0476 \mathrm{GHz}$ and $\omega_{2}=55.3674 \mathrm{GHz}$, taken from [26], at room temperature, with equal loss rates $\gamma=100 \mathrm{kHz}$.

In Fig. 4 we plot the difference between the logarithmic negativity obtained with repeated control operations and the one obtained with a single initial control. We show that applying more control operations after the initial one delays the quantitative decay of entanglement of a small but not altogether negligible amount; interestingly, the sudden death of entanglement is only very slightly delayed by applying further control operations. These results are only a particular example of the same general behavior we found from similar numerical analyses. However, we also found that the effect of repeated control operation is more marked when it is acting on only one mode.

To illustrate that interspersed, time-local optimal control actions might fail to optimize globally, let us report on a peculiar case, with normal form parameters $a=5, b=6$, $c_{+}=5.2$, and $c_{-}=-4.8$, in Fig. 5 , where we plot the difference between the logarithmic negativity obtained with repeated control operations and the one where no controls are applied and the initial state in normal form remains in normal form throughout the evolution. We see that applying the control on both modes always provides one with an increased logarithmic negativity and that, as shown before, controlling more than once gives little improvements. On the other hand, optimized control operations on a single mode show that, whilst initially offering a certain advantage in terms of logarithmic negativity, can actually make it decrease sooner 


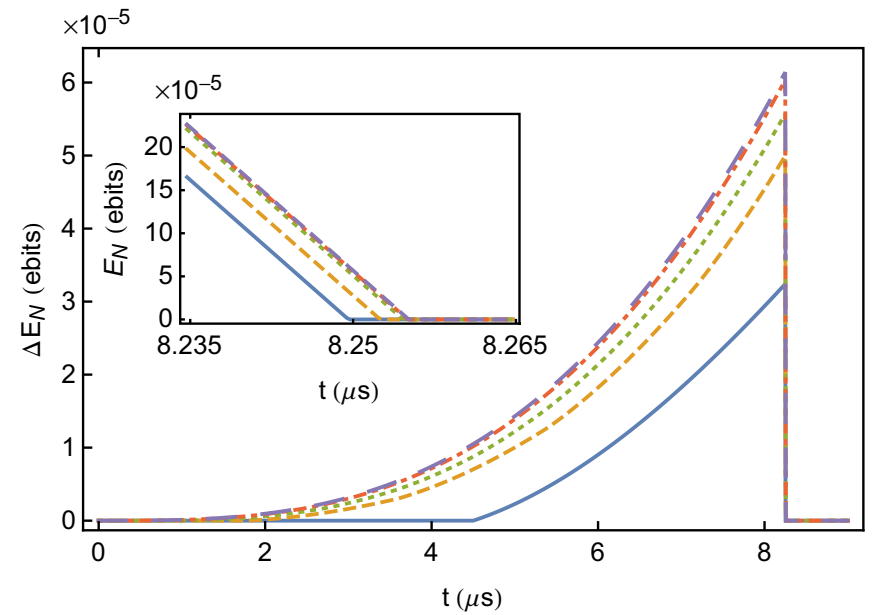

FIG. 4. Difference between the logarithmic negativity with repeated controls and the one with a single initial control, for an initial state in normal form with parameters $a=4.5, b=3.5, c_{+}=2.2$, and $c_{-}=-3.5$, evolving in thermal environments with $\chi_{1} / \gamma=$ $1.14769, \chi_{2} / \gamma=1.02956$, and same loss rate $\gamma=100 \mathrm{kHz}$. The blue (solid) curve represents one additional control operation after the first one, the orange (short dashed) curve three additional control operations, the green (dotted) curve represents a total of ten control operations, and the red (dot-dashed) curve represents a total of 100 control operations, while the purple (large dashed) curve is obtained when the control is applied at every time step. Inset: the slight delay of entanglement "sudden death" due to repeated controls. The time step used in the plots is $2 \times 10^{-3} \mu$ s and $3 \times 10^{-4} \mu$ s for the main plot and the inset, respectively.

than without any control operations. It is also apparent that in this case repeated control operations have a more marked effect.

\section{ALTERNATIVE LOCAL OPTIMIZATION}

As previously said, the control procedures we identified in the previous sections do not necessarily achieve a global optimization of logarithmic negativity. It is therefore interesting to explore alternative locally optimal controls and compare them to what we achieved above. As one such alternative, we shall consider the time-local minimization of the quantity $\tilde{\Sigma}=$ Det $\sigma-\tilde{\Delta}_{1}^{2}+1$ whose negativity is a necessary and sufficient signature of entanglement; see Eq. (B1) in Appendix B.

One simply has $\dot{\tilde{\Sigma}}=\operatorname{Det} \sigma-\dot{\tilde{\Delta}}_{1}^{2}$, where the two terms are the ones found previously in Eqs. (21) and (22). This leads to a quantity depending on the control parameters $z_{1}$ and $z_{2}$, which will be termed $\quad \xi^{\prime}=\chi_{1}\left(\operatorname{Det} \boldsymbol{\beta} \operatorname{Tr}\left[S_{1}(\boldsymbol{\sigma} / \boldsymbol{\beta}) S_{1}^{\top}\right]-\operatorname{Tr}\left[S_{1} \boldsymbol{\alpha} S_{1}^{\top}\right]\right)+$ $\chi_{2}\left(\operatorname{Det} \boldsymbol{\alpha} \operatorname{Tr}\left[S_{2}(\boldsymbol{\sigma} / \boldsymbol{\alpha}) S_{2}^{\top}\right]-\operatorname{Tr}\left[S_{2} \boldsymbol{\beta} S_{2}^{\top}\right]\right)$. Explicitly, we are interested in the minimization of

$$
\xi^{\prime}=\chi_{1}\left(v_{1}^{\prime} z_{1}^{2}+\frac{w_{1}^{\prime}}{z_{1}^{2}}\right)+\chi_{2}\left(v_{2}^{\prime} z_{2}^{2}+\frac{w_{2}^{\prime}}{z_{2}^{2}}\right),
$$

with

$$
\begin{aligned}
& v_{1}^{\prime}=b\left(a b-c_{+}^{2}\right)-a, \\
& w_{1}^{\prime}=b\left(a b-c_{-}^{2}\right)-a,
\end{aligned}
$$

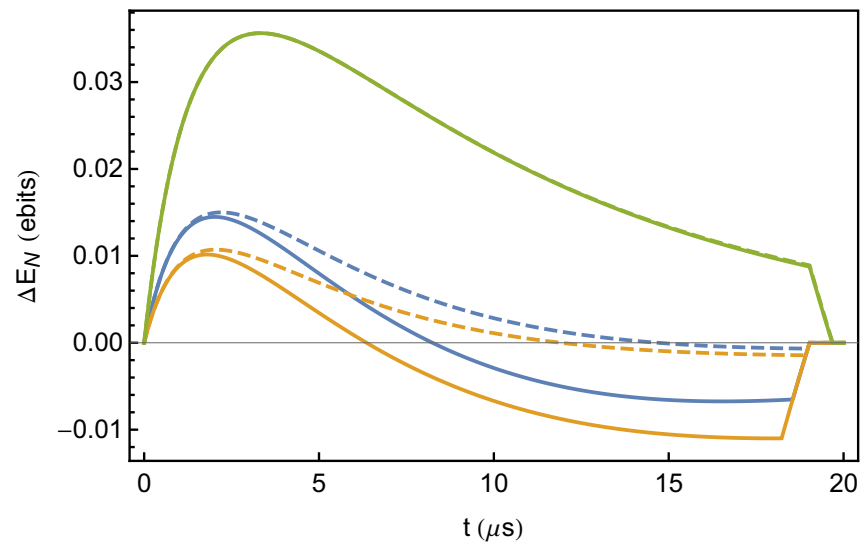

FIG. 5. Difference between the logarithmic negativity with repeated controls and the one without control, for an initial state with normal form parameters $a=5, b=6, c_{+}=5.2$, and $c_{-}=$ -4.8 , evolving in two thermal environments with $\chi_{1} / \gamma=1.14769$, $\chi_{2} / \gamma=1.02956$, and the same loss rate $\gamma=100 \mathrm{kHz}$. For each color, the solid lines always represent the application of an initial control operation, while the dashed curves represent controls applied at each time of the evolution (the time step chosen for the plot is $\left.10^{-2} \mu \mathrm{s}\right)$. The green curves above all the others represent control operations on both modes and they are practically superimposed at this scale, the dashed one attaining slightly higher values of $\Delta E_{\mathcal{N}}$. The blue curves in the middle (in the region $t \approx 2 \mu \mathrm{s}$ ) represent a control operation on subsystem 1 , while the orange curves at the bottom (again, in the region $t \approx 2 \mu \mathrm{s}$ ) pertain to control operations on subsystem 2 only.

$$
\begin{aligned}
& v_{2}^{\prime}=a\left(a b-c_{+}^{2}\right)-b, \\
& w_{2}^{\prime}=a\left(a b-c_{-}^{2}\right)-b,
\end{aligned}
$$

which is achieved for the choice

$$
z_{j}=\sqrt[4]{\frac{w_{j}^{\prime}}{v_{j}^{\prime}}}
$$

This optimization, at variance with the one detailed in Sec. III, requires a nontrivial control on the fly to be enforced at every instant in time, for every choice of initial states and dynamics (even symmetric ones).

However, rather interestingly, a direct numerical comparison reveals that this control criterion is not globally optimal in order to minimize $\tilde{\Sigma}$, as shown in a specific case in Fig. 6 . In general, the control minimizing $\tilde{v}_{-}$is always superior in delaying the disappearance of entanglement in a thermal environment, although the difference between the two methods is marginal in the region around disentanglement [32]. In the case depicted in Fig. 6 of an initial state in normal form with $a=4.5, b=3.5, c_{+}=2.2$, and $c_{-}=-3.5$ evolving in an environment with thermal noise $\chi / \gamma=2$ and loss rate $\gamma=1 \mathrm{MHz}$, the disappearance of entanglement is delayed by the optimal control method by only $4 \mathrm{~ns}$ with respect to the method minimizing $\tilde{\Sigma}$ (9486 ns against 9482 ns). Note that the difference involved is well detected by our numerical precision. 


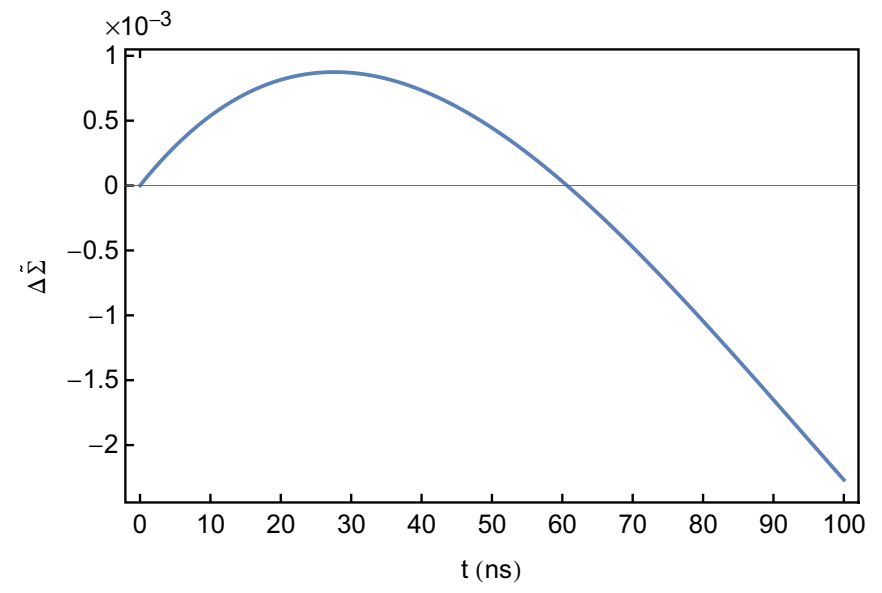

FIG. 6. Difference in the separability parameter $\tilde{\Sigma}$ between optimal local control minimizing $\tilde{v}_{-}$and optimal local control minimizing $\tilde{\Sigma}$ for an initial state in normal form with $a=4.5, b=3.5, c_{+}=$ 2.2 , and $c_{-}=-3.5$ evolving in an environment with equal thermal noises with $\chi / \gamma=2$ and loss rates $\gamma=1 \mathrm{MHz}$. As apparent, after about $30 \mathrm{~ns}$, the local minimization of $\tilde{v}_{-}$offers an advantage also in minimizing $\tilde{\Sigma}$, whose time-local minimization is thus shown to be globally suboptimal. The evolving state is entangled up to a time of around $90 \mathrm{~ns}$.

We have thus encountered an instance where the time-local control of a parameter is not globally optimal. The timelocal minimization of $\tilde{v}_{-}$, offering the additional advantage of requiring a single initial manipulation, is certainly to be preferred to a control minimizing $\tilde{\Sigma}$. Indeed, a broad numerical exploration could identify no procedure that would surpass the time-local minimization of $\tilde{v}$ in maximizing the final entanglement, although we do not possess an analytical proof of such optimality.

\section{SUMMARY AND OUTLOOK}

Summarizing, we have identified a local manipulation through unitary operations, comprised of the local transformations that put the state in standard form followed by local squeezing transformations with specific parameters, that guarantee that a two-mode Gaussian state evolving in two generic, and generally different, thermal environments (one for each mode) will experience the minimal possible loss of logarithmic negativity at each moment in time. Very remarkably, for states with symmetric correlations and for the same dynamics on both modes, the optimal scheme only involves an initial local unitary adjustment of the state, with no subsequent action. Interestingly, even when the initial preparation alone is not optimal, we find that the results are quantitatively very close to those obtained with continuous, on-the-fly control. Such a strategy has been also compared with alternative control methods (optimizing different figures of merit) and found to be superior. It is worth mentioning that the optimal transformation to be applied does not depend at all on the parameters of the noisy evolution. This advance in the understanding of the manipulation and control of continuousvariable entanglement, besides its inherent theoretical relevance, would be applicable to systems of direct and imme- diate experimental interest $[11,12]$. Let us also remark that the analysis we carried out straightforwardly extends to all bipartite multimode Gaussian states which are invariant under permutation of the modes within any one partition, since such states are locally equivalent to two-mode states [33]; notably, this includes all 1 vs $n$-mode systems.

It is also worth mentioning that the ability to perform instantaneous operations on the system would in principle allow one to employ dynamical decoupling techniques [34], whereby the system could be entirely decoupled from its environment, thus preserving its entanglement. Such a method, while mostly studied for finite-dimensional systems, can also be implemented in the continuous-variable regime [35], as recently shown in full generality for quadratic Hamiltonians in [36]. It does, however, require one to perform control operations at a rate which is much faster than the environment cutoff frequency, which is conceivable in certain scenarios but impractical in optical setups. Quite on the contrary, our strategies turn out to rely in general on very few control pulses (often on a single initial one).

In the future, this study could be extended to consider a different set of instantaneous operations, consisting of passive unitaries but without the restriction to being local, i.e., to the optimization of entanglement through repeated beam splitters [one can already see from Eqs. (21) and (22) that phase shifters would be useless to the task]. The interest of such a problem lies in the fact that in quantum optics passive optical elements are generally considered to be freely available resources $[37,38]$, while squeezing operations are the expensive ingredient needed to create entanglement.

Yet another worthwhile extension of the present work would consist in adding an interaction Hamiltonian between the two modes during the dynamics. Since the interaction has the potential to create entanglement, it would be interesting to study its interplay with local controls, possibly on only one of the modes. This setting will be particularly relevant to optomechanical systems, where the interaction Hamiltonian is key to, e.g., sideband driving, and the thermal noise on the mechanical mode tends to suppress nonclassical features.

\section{ACKNOWLEDGMENT}

We thank D. Burgarth for bringing analogous studies based on dynamical decoupling to our attention.

\section{APPENDIX A: SYMPLECTIC INVARIANTS}

All the spectral properties of Gaussian states are clearly invariant under unitary, and hence symplectic, transformations, and must thus be determined by the symplectic invariants of the covariance matrix [30,39]. The normal mode decomposition, that allows one to turn any covariance matrix $\sigma$ into normal form through some symplectic transformation $S$, as per $S \sigma S^{\top}=\bigoplus_{j=1}^{n} v_{j} \mathbb{1}$, shows that $n$ such independent invariants can be constructed from an $n$-mode covariance matrix. A possible choice of symplectic invariants is represented by the $n$ quantities $v_{j}$, known as the symplectic eigenvalues, that may be determined as the moduli of the eigenvalues of the matrix $\Omega \sigma$ which, due to the symmetry of $\sigma$ and antisymmetry 
of $\Omega$, come in degenerate pairs. In terms of the symplectic eigenvalues, the uncertainty relation reads $v_{j} \geqslant 1$.

The information contained in the symplectic eigenvalues may be expressed in terms of other sets of invariants, such as the sums of the $2 k \times 2 k$ principal minors of the matrix $\Omega \sigma$, which we shall denote with $\Delta_{k}^{n}$. Up to a sign, these correspond to the coefficients of the characteristic polynomial of $\Omega \sigma$. It turns out that the uncertainty relation implies [30]

$$
\Sigma=\sum_{k=0}^{n}(-1)^{n+k} \Delta_{k}^{n} \geqslant 0
$$

to be read with the additional stipulation $\Delta_{0}^{n}=1$. For twomode states, the independent invariants are the determinant $\operatorname{Det} \sigma=\Delta_{2}^{2}$ and the quantity $\Delta_{1}^{2}=\operatorname{Det} \boldsymbol{\alpha}+\operatorname{Det} \boldsymbol{\beta}+2 \operatorname{Det} \boldsymbol{\gamma}$ [40], for the $2 \times 2$ submatrices $\boldsymbol{\alpha}, \boldsymbol{\beta}$, and $\boldsymbol{\gamma}$ of Eq. (4).

\section{APPENDIX B: ENTANGLEMENT OF GAUSSIAN STATES}

The properties related to the entanglement of Gaussian states are instead determined by quantities that are invariant under local symplectic transformations. Since the positivity of the partial transposition is necessary and sufficient for the separability of 1 vs $n$ and locally symmetric Gaussian states, it turns out that a satisfactory characterization of the entanglement of such states, which encompass all two-mode states, is achieved in terms of partially transposed symplectic eigenvalues and invariants. At the level of covariance matrices, the partial transposition $\tilde{\sigma}$ of the first $n_{A}$ modes is described by the congruence transformation $\tilde{\sigma}=T \sigma T$ for $T=\left(\bigoplus_{j=1}^{n_{A}} \sigma_{z}\right) \oplus$ $\mathbb{1}_{2\left(n-n_{A}\right)}$, where $\sigma_{z}$ is the Pauli matrix $\operatorname{diag}(1,-1)$ and $\mathbb{1}_{2\left(n-n_{A}\right)}$ is the identity matrix on the remaining $\left(n-n_{A}\right)$ modes. For this set of Gaussian states, the violation of the inequality $\tilde{\sigma}+i \Omega$ is necessary and sufficient for entanglement. In terms of the sums of principal minors of $\Omega \tilde{\sigma}$, denoted with $\tilde{\Delta}_{k}^{n}$, one has the corresponding necessary and sufficient separability condition

$$
\tilde{\Sigma}=\sum_{k=0}^{n}(-1)^{n+k} \tilde{\Delta}_{k}^{n} \geqslant 0 .
$$

An equivalent necessary and sufficient condition for separability may be written down in terms of the smallest partially transposed symplectic eigenvalue of $\sigma$, denoted with $\tilde{v}_{-}$:

$$
\tilde{v}_{-} \geqslant 1 \text {. }
$$

For the class of states in hand, at most one partially transposed symplectic eigenvalue may be smaller than 1 [30]. In turn, $\tilde{v}_{-}$ determines the state's logarithmic negativity $E_{\mathcal{N}}$, as

$$
E_{\mathcal{N}}=\max \left\{0,-\log _{2}\left(\tilde{v}_{-}\right)\right\} .
$$

The quantity $E_{\mathcal{N}}$ sets an upper bound, expressed in entangled bits (ebits), to the distillable entanglement, i.e., the rate of maximally entangled pairs of qubits that can be distilled through local operations and classical communication in the asymptotic limit of an infinite number of available copies $[29,41,42]$. This is the entanglement quantifier that we shall adopt as a figure of merit for our control scheme.

For two-mode states, the partially transposed invariants are the determinant Det $\sigma$ which, by virtue of Binet's theorem, is not affected by the partial transposition $T$, and $\tilde{\Delta}_{1}^{2}=\operatorname{Det} \boldsymbol{\alpha}+$
Det $\boldsymbol{\beta}-2 \operatorname{Det} \boldsymbol{\gamma}$; notice the minus sign that distinguishes this quantity from $\Delta_{1}^{2}$. This yields the separability criterion

$$
\tilde{\Sigma}=\operatorname{Det} \sigma-\tilde{\Delta}_{1}^{2}+1 \geqslant 0,
$$

and the smallest partially transposed symplectic eigenvalue $\tilde{v}_{-}$ determined by the relationship

$$
2 \tilde{\nu}_{-}^{2}=\tilde{\Delta}_{1}^{2}-\sqrt{\left(\tilde{\Delta}_{1}^{2}\right)^{2}-4 \operatorname{Det} \sigma} .
$$

\section{APPENDIX C: EQUATIONS OF MOTION FOR THE SYMPLECTIC INVARIANTS OF TWO-MODE STATES}

Given the block form (4), we explicitly write the equation of motion for the covariance matrix (9) of a two-mode state

$$
\dot{\boldsymbol{\sigma}}=\left(\begin{array}{cc}
\dot{\boldsymbol{\alpha}} & \dot{\boldsymbol{\gamma}} \\
\dot{\boldsymbol{\gamma}}^{\top} & \dot{\boldsymbol{\beta}}
\end{array}\right)=\left(\begin{array}{cc}
-\gamma_{1} \boldsymbol{\alpha}+\chi_{1} \mathbb{1} & -\frac{\gamma_{1}+\gamma_{2}}{2} \boldsymbol{\gamma} \\
-\frac{\gamma_{1}+\gamma_{2}}{2} \boldsymbol{\gamma}^{\top} & -\gamma_{2} \boldsymbol{\beta}_{2}+\chi_{2} \mathbb{1}
\end{array}\right),
$$

as well as the solution $\sigma(t)$ in terms of its submatrices:

$$
\begin{array}{r}
\boldsymbol{\alpha}(t)=\frac{\chi_{1}}{\gamma_{1}} \mathbb{1}+\left(\boldsymbol{\alpha}(0)-\frac{\chi_{1}}{\gamma_{1}} \mathbb{1}\right) e^{-\gamma_{1} t}, \\
\boldsymbol{\gamma}(t)=\boldsymbol{\gamma}(0) e^{-\frac{\gamma_{1}+\gamma_{2}}{2} t}, \\
\boldsymbol{\beta}(t)=\frac{\chi_{2}}{\gamma_{2}} \mathbb{1}+\left(\boldsymbol{\beta}_{2}(0)-\frac{\chi_{2}}{\gamma_{2}} \mathbb{1}\right) e^{-\gamma_{2} t} .
\end{array}
$$

A key step in our analysis is to determine the equations that govern the evolution of the symplectic invariants under this dynamics. which may be obtained through Jacobi's formula: $\frac{d}{d t} \operatorname{Det} A(t)=\operatorname{Tr}\left[\operatorname{Adj}(A(t)) \frac{d}{d t} A(t)\right]$, where $\operatorname{Adj}(A(t))$ represents the adjugate matrix of $A(t)$; for invertible matrices we have $\operatorname{Adj}(A(t))=(\operatorname{Det} A(t)) A(t)^{-1}$. For two modes the equations for the two invariants of the partially transposed state $\tilde{\Delta}_{2}^{2}=\operatorname{Det} \sigma$ and $\tilde{\Delta}_{1}^{2}$ can be explicitly written as

$$
\begin{aligned}
\operatorname{Det} \boldsymbol{\sigma}= & (\operatorname{Det} \boldsymbol{\sigma}) \operatorname{Tr}\left[\boldsymbol{\sigma}^{-1} \dot{\boldsymbol{\sigma}}\right] \\
= & -2\left(\gamma_{1}+\gamma_{2}\right) \operatorname{Det} \boldsymbol{\sigma} \\
& +\chi_{1} \operatorname{Det} \boldsymbol{\beta} \operatorname{Tr}[\boldsymbol{\sigma} / \boldsymbol{\beta}]+\chi_{2} \operatorname{Det} \boldsymbol{\alpha} \operatorname{Tr}[\boldsymbol{\sigma} / \boldsymbol{\alpha}], \\
\dot{\tilde{\Delta}}_{1}^{2}= & \frac{d}{d t}(\operatorname{Det} \boldsymbol{\alpha}+\operatorname{Det} \boldsymbol{\beta}-2 \operatorname{Det} \boldsymbol{\gamma}) \\
= & -2 \gamma_{1} \operatorname{Det} \boldsymbol{\alpha}-2 \gamma_{2} \operatorname{Det} \boldsymbol{\beta}+2\left(\gamma_{1}+\gamma_{2}\right) \operatorname{Det} \boldsymbol{\gamma} \\
& +\chi_{1} \operatorname{Tr} \boldsymbol{\alpha}+\chi_{2} \operatorname{Tr} \boldsymbol{\beta},
\end{aligned}
$$

where we used the time derivative of the submatrices (C1), formulas for the inverse of a block matrix, and the fact that $\operatorname{Tr}[\operatorname{Adj}(A)]=\operatorname{Tr} A$ for $2 \times 2$ matrices [to obtain Eq. (C5), we also assumed Det $\boldsymbol{\gamma} \neq 0$, because a multiplication by $\boldsymbol{\gamma}^{-1}$ is needed: this is always possible since Det $\boldsymbol{\gamma}<0$ is a necessary condition to have a nonseparable state]. The two matrices $\boldsymbol{\sigma} / \boldsymbol{\beta}=\boldsymbol{\alpha}-\boldsymbol{\gamma} \boldsymbol{\beta}^{-1} \boldsymbol{\gamma}^{\top}$ and $\boldsymbol{\sigma} / \boldsymbol{\alpha}=\boldsymbol{\beta}-\boldsymbol{\gamma}^{\top} \boldsymbol{\alpha}^{-1} \boldsymbol{\gamma}$ are the Schur complements of $\boldsymbol{\sigma}$ with respect to the submatrices $\boldsymbol{\beta}$ and $\boldsymbol{\alpha}$.

The relationships (C5) and (C6) form the basis of our analysis for the control of entanglement. Notice that, on the right-hand side, whilst all the determinants are local 
symplectic invariants and are thus unaffected by local symplectic transformations, all the traces that multiply the coef- ficients $\chi_{1}$ and $\chi_{2}$ are not. These terms identify the handle through which local symplectic control may act.
[1] J. M. Raimond, M. Brune, and S. Haroche, Rev. Mod. Phys. 73, 565 (2001).

[2] V. S. Malinovsky and I. R. Sola, Phys. Rev. A 70, 042304 (2004).

[3] S. Mancini and H. M. Wiseman, Phys. Rev. A 75, 012330 (2007).

[4] N. Yamamoto, H. I. Nurdin, M. R. James, and I. R. Petersen, Phys. Rev. A 78, 042339 (2008).

[5] A. Serafini and S. Mancini, Phys. Rev. Lett. 104, 220501 (2010).

[6] H. I. Nurdin and N. Yamamoto, Phys. Rev. A 86, 022337 (2012).

[7] C. E. Creffield, Phys. Rev. Lett. 99, 110501 (2007).

[8] A. R. R. Carvalho and J. J. Hope, Phys. Rev. A 76, 010301 (2007).

[9] A. R. R. Carvalho, A. J. S. Reid, and J. J. Hope, Phys. Rev. A 78, 012334 (2008).

[10] R. N. Stevenson, J. J. Hope, and A. R. R. Carvalho, Phys. Rev. A 84, 022332 (2011).

[11] J. Laurat, G. Keller, J. A. Oliveira-Huguenin, C. Fabre, T. Coudreau, A. Serafini, G. Adesso, and F. Illuminati, J. Opt. B 7, S577 (2005).

[12] F. A. S. Barbosa, A. S. Coelho, A. J. de Faria, K. N. Cassemiro, A. S. Villar, P. Nussenzveig, and M. Martinelli, Nat. Photon. 4, 858 (2010).

[13] Z. Yan, X. Jia, C. Xie, and K. Peng, Phys. Rev. A 84, 062304 (2011).

[14] G. Masada, K. Miyata, A. Politi, T. Hashimoto, J. L. O’Brien, and A. Furusawa, Nat. Photon. 9, 316 (2015).

[15] L. Martin, M. Sayrafi, and K. B. Whaley, Quantum Sci. Technol. 2, 044006 (2017).

[16] X. Deng, C. Tian, X. Su, and C. Xie, Sci. Rep. 7, 44475 (2017).

[17] C. P. Koch, J. Phys.: Condens. Matter 28, 213001 (2016).

[18] A. Serafini, Quantum Continuous Variables: A Primer of Theoretical Methods (CRC Press, Boca Raton, 2017).

[19] V. Mukherjee, A. Carlini, A. Mari, T. Caneva, S. Montangero, T. Calarco, R. Fazio, and V. Giovannetti, Phys. Rev. A 88, 062326 (2013).

[20] A. Carlini, A. Mari, and V. Giovannetti, Phys. Rev. A 90, 052324 (2014).
[21] U. Shackerley-Bennett, A. Carlini, V. Giovannetti, and A. Serafini, Quantum Sci. Technol. 2, 044014 (2017).

[22] A. Serafini, S. D. Siena, F. Illuminati, and M. G. A. Paris, J. Opt. B 6, S591 (2004).

[23] A. Serafini, M. G. A. Paris, F. Illuminati, and S. De Siena, J. Opt. B 7, R19 (2005).

[24] R. Filip, Phys. Rev. A 87, 042308 (2013).

[25] H. Le Jeannic, A. Cavaillès, K. Huang, R. Filip, and J. Laurat, Phys. Rev. Lett. 120, 073603 (2018).

[26] E. Flurin, N. Roch, F. Mallet, M. H. Devoret, and B. Huard, Phys. Rev. Lett. 109, 183901 (2012).

[27] R. Simon, Phys. Rev. Lett. 84, 2726 (2000).

[28] U. Shackerley-Bennett, A. Pitchford, M. G. Genoni, A. Serafini, and D. K. Burgarth, J. Phys. A 50, 155203 (2017).

[29] M. B. Plenio, Phys. Rev. Lett. 95, 090503 (2005).

[30] A. Serafini, Phys. Rev. Lett. 96, 110402 (2006).

[31] G. Adesso, A. Serafini, and F. Illuminati, Phys. Rev. A 70, 022318 (2004).

[32] That one of such two inequivalent methods should be suboptimal globally could be inferred, barring the case that they would lead to the very same disentanglement time, by the fact that both the condition $\tilde{\Sigma}<0$ and $\tilde{v}_{-}<1$ are necessary and sufficient for entanglement.

[33] L. Lami, A. Serafini, and G. Adesso, New J. Phys. 20, 023030 (2018).

[34] L. Viola, E. Knill, and S. Lloyd, Phys. Rev. Lett. 82, 2417 (1999).

[35] D. Vitali and P. Tombesi, Phys. Rev. A 59, 4178 (1999).

[36] C. Arenz, D. K. Burgarth, and R. Hillier, J. Phys. A: Math. Theor. 50, 135303 (2017).

[37] K. C. Tan, T. Volkoff, H. Kwon, and H. Jeong, Phys. Rev. Lett. 119, 190405 (2017).

[38] B. Yadin, V. Narasimhachar, F. C. Binder, J. Thompson, M. Gu, and M. S. Kim, arXiv:1804.10190.

[39] V. V. Dodonov, J. Phys. A 33, 7721 (2000).

[40] A. Serafini, F. Illuminati, and S. De Siena, J. Phys. B 37, L21 (2004).

[41] G. Vidal and R. F. Werner, Phys. Rev. A 65, 032314 (2002).

[42] J. Lee, M. S. Kim, Y. J. Park, and S. Lee, J. Mod. Opt. 47, 2151 (2000). 